\title{
Awareness and Perception of Agile in Saudi Software Industry
}

\author{
Fahad S Altuwaijri ${ }^{1,2}$ and Maria Angela Ferrario ${ }^{1,3}$ \\ ${ }^{1}$ School of Computing and Communications, Lancaster University, UK \\ $\{$ f.altuwaijri,m.ferrario\}@lancaster.ac.uk \\ ${ }^{2}$ Department of Information Technology, College of Computer, Qassim University, Buraydah, Saudi Arabia \\ f.altuwaijri@qu.edu.sa \\ ${ }^{3}$ School of Electronics, Electrical Engineering and Computer Science, Queen's University, Belfast, UK \\ m.ferrario@qub.ac.uk
}

\begin{abstract}
Over the last two decades, Agile software development methodology has garnered significant interest in both software industry and research with several studies investigating the level of awareness, perception and use of Agile in software engineering practice. However, most of these studies have focused on Agile practice in developed countries, with only a handful having been conducted in developing countries, especially in the Middle East. This is problematic given the strategic and economic importance of software industry in countries such as Saudi Arabia. This paper aims to start addressing this research gap with an empirical investigation of the awareness and perceptions of Agile among software practitioners in Saudi Arabia and the extent to which they are adopting Agile methods. To this end, we conduct four semi-structured interviews with expert software practitioners and a survey with 31 respondents, all from the mobile development software industry. Our findings indicate that there it seems to be a low level of awareness and usage of Agile in the country. However, we also find that those who use Agile, are appreciative of its benefits which include project management flexibility, rapid response to change, and a positive effect on team morale and communication.
\end{abstract}

Index Terms-Agile software development, Agile methods, Agile, mobile application development, mobile apps, software engineering, Awareness, Perception

\section{INTRODUCTION}

In recent years, software development management has become an extremely complex task. Due to the dynamic nature of the software market and frequently changing customers' needs, companies must respond quickly to change to stay competitive [1]. To this end, organisations are exploring new approaches to software project management by improving time to market, reducing costs and improving quality. In particular, Agile software development has brought a significant change to software development by providing an iterative and incremental approach and the use of different methods such as Scrum, Crystal and Extreme Programming, which respond to customers' changing needs in a rapid and flexible manner [2].

Although Agile values and principles underpin software engineering practice across the world, it is worth noting that its roots are quintessentially western. The very birth of the Agile Manifesto happened in a US ski resort, where a fairly culturally homogenous group of software practitioners met and introduced the Agile to the world [3]. With this observation, we mean not to detract the importance of Agile, instead we wish to draw the attention to possible values' differences that may affect and shape Agile adoption in countries with different values orientations [4], [5]. Thus, organisations need to develop culturally aware approaches to support their teams to use Agile.

The awareness and perception of Agile among software practitioners have attracted much research over the years to understand to what extent Agile can be adopted and used. However, most studies have focused on the practice in developed countries [6]-[9], with only a handful being carried out in developing countries [10]-[12] and no research conducted in Middle Eastern countries, particularly in Saudi Arabia. We find this problematic on two accounts: scientifically and strategically. Firstly, Agile is a values-based and principled approach to software development, which, as explained above, emanated from a rather homogeneous western worldview of software development. Secondly, in recent years, developing countries such as Saudi Arabia have been heavily investing in the software industry sector, seen as a strategic direction for diversifying the economy beyond the oil industry. Therefore, neglecting a geographic area where the software industry is rapidly growing seems to be strategically limited.

This paper investigates the awareness, usage and perception of Agile among Saudi software practitioners through empirical research. Specifically, this study focuses on mobile development practice, which research has found to be particularly suited to Agile [6], [13]-[15]. The objectives of this study are to understand 1) the level of awareness and current usage of Agile in the Saudi mobile software industry; 2) the reasons for adopting and not adopting Agile, and 3) software practitioner perceptions of Agile methods.

In terms of approach, this work is an empirical study and adopts a mixed-method approach to achieve its aims. First, expert interviews were conducted with four experts in mobile and Agile development to explore their viewpoints about the awareness and perceptions of Agile and to use their feedback in order to design the second method of the study. Second, a survey was then conducted to identify awareness and perceptions of software development methodologies and Agile, which received 31 participants. This study makes contributions 
to the body of knowledge of Agile adoption in the Saudi software community by providing broad insights on current Agile use and may also be used to increase awareness of Agile in the country. In addition, it can be used as a roadmap for further investigations.

The rest of the paper is organised as follow: Section II reviews the related work. Section III describes the research methods adopted in this study, while the results of the data collected are presented in Section IV and Section V. The discussion of these results is discussed in Section VI. Finally, Section VII concludes the work, presents its limitations, and put forward some suggestions for future work.

\section{RELATED WORK}

Understanding practitioners' awareness and perceptions of Agile is considered an essential step in investigating the factors influencing Agile adoption, and several research efforts have been reported in this area [8]-[12], [16]-[19]. These studies investigate the level of awareness of Agile amongst software practitioners, their current usage, and perceptions towards Agile through empirical research. Their findings revealed that Agile has a positive impact on the software development process. An example of these studies is the work of Rodriguez et al. [7] who studied the impact of Agile usage in Finnish software industry through a survey with 408 responses. The findings of this study indicate that adopting Agile can increase the productivity and the products' quality as well as accelerate time-to-market.

The majority of these studies have been conducted in Agile industry in developed countries (e.g. Rodríguez et al. [7]; Begel and Nagappen [8]; Solinski and Petersen [17]; Salo and Abrahamson [9]), and only a handful conducted in developing countries (e.g. Melo et al. [10]; Salinas, Neto and Emer [16]; Nazir, Hasteer and Bansal [11]; Sulaiman, Mahrin and Yusoff [12]; Nanthaamornphong and Wetprasit [20]). However, none of these studies focused on the software industry in Middle Eastern countries. This is problematic, specifically in the case of Saudi Arabia, which is increasingly looking at the software industry to diversify its economy to reduce the reliance on the oil industry.

For instance, Begel and Nagappen [8] conducted an investigation into the use and perception of Agile among Microsoft employees across three continents (i.e. North America, Asia and Europe), however, it only concentrated on one company, and one that has a distinctly western cultural orientation. Their study revealed that $33 \%$ of the developers working in Microsoft use Agile and they have a positive opinion towards it. The study focused on Microsoft's organisational culture and did not discuss the differences in Agile adoption and perception across the regions.

Melo et al. [10] investigated the evolution of Agile and its adoption in the Brazilian community by conducting a survey, had 471 responses, and seven semi-structured interviews. They found that a lack of documentation is the primary concern for practitioners when adopting Agile, and that organisational culture change is one of the main challenges in the process of adopting Agile.

Bin-Hezam et al. [21] examined to what extent Agile has been adopted by SMEs in Saudi Arabia from a variety of industry sectors. The authors distributed a survey that received 26 responses to collect data for their study. The findings of the study show that although many companies have adopted Agile, most of them are not aware of Agile practices.

In general, very little studies have been done around Agile industry context and particularly in mobile app development domain, where a few studies happen in academic context such as the study of Santos et al. [6], which investigated Agile perception and adoption of Agile in mobile app development. Its findings show that the adoption of Agile in developing mobile apps means that it not only reduces the amount of risks that are associated with it, but also provides better management and control and allows developers enough flexibility and freedom to develop a mobile application that has the ability to adjust to changes quickly.

Therefore, previous studies have focused on investigating the awareness, current usage and perceptions of Agile almost exclusively in developed countries. This research aims to reorient the focus of the studies on emerging economies, using Saudi Arabia as a case study.

\section{RESEARCH METHOD}

This study adopted a mixed-method approach, as recommended by Creswell [22] and Biesta et al. [23], adopting this approach to research enables researchers to gain more knowledge and increase the reliability of the findings. The presented study is hence divided into two phases: qualitative and quantitative. Interviews with experts were conducted during the first phase $(\mathrm{N}=4)$, and then a survey was carried out in the second phase of the study $(\mathrm{N}=31)$. Ethical approval was sought and obtained from the authors' institution following the standard procedures required.

\section{A. First Phase: Interview}

The data were collected from semi-structured interviews. During the interview sessions, closed- and open-ended questions were asked. The interviews were conducted online with four experts who have many years' industrial experience and knowledge of both mobile app development and Agile software development. These experts worked at different organisations in the sector of mobile app development in Saudi Arabia. The aim of the interviews was to examine the experts' views of the general awareness and perception of Agile in Saudi Arabia. The input gathered during this phase was then used to guide the development of the survey used in the second phase.

The interview questions were divided into two sections, and each session lasted for about 30-60 minutes; in total, about 180 minutes of recorded material. In the first section, demographic information was collected to examine the interviewees' background and their organisations' details. In the second section, 
interviewees were asked to discuss their awareness and perceptions of Agile from their point of view. Interviews were audio-recorded with the interviewees' permission and notes were also taken. All interview data were transcribed and then organised and coded using the thematic analysis approach [24], [25] with the support of NVivo software. The transcriptions, codes and themes were performed by the first author and then discussed with the second author.

\section{B. Second Phase: Survey}

The second data collection method involved a web-based survey, which also aimed to identify the participants' awareness and perception of software development methodologies, particularly Agile software development in Saudi Arabia. 31 responses were received from the targeted respondents who were mobile app practitioners in Saudi Arabia, who have either adopted or not Agile methods.

A cross-sectional web-based survey was selected for use in this research and created by one of many online survey tools (i.e. Qualtrics). The survey was comprised of 26 questions and consisted of three parts. The first part gathered demographic data relating to the participants and their work context. The second part focused on software development, while in the third part, the participants were asked to share their views on Agile and their perception of it. Multiple-choice questions and 5-point Likert scales were used to reflect the points of view of the respondents. In question 12, the participants were asked about their knowledge of Agile, and if they selected the 'Not known' option, they were able to proceed directly to answer the questions from number 24, which were about the use of Agile techniques, and then continue on to complete the survey.

A pilot study was conducted to test the readability and validity of the questions. Two $\mathrm{PhD}$ candidates and two mobile app practitioners from Saudi Arabia participated in this pilot study. Several amendments were made to the items before the survey was distributed, based on their feedback. The sets of survey were distributed to mobile app practitioners using a snowball sampling approach [26], and were distributed through email and on social media. The data were collected for four weeks and were analysed using statistical software (i.e. SPSS). This determines the relationships and trends in the data and presents them in the form of graphs and cross-tabulated formats [27].

\section{INTERVIEW FINDINGS}

This section reports the results of the qualitative semistructured interviews with four mobile app development and Agile software development experts from Saudi Arabia to obtain a deeper understanding of the awareness and perception of Agile in Saudi Arabia.

\section{A. Demographics and Work Information}

Table I summarises the demographic information of the experts and their work context. Each interviewee was given a pseudonym to conceal their identity and the name of their organisations. Each interviewee had a different role in their organisations, including one $\mathrm{CEO}$, one CTO, one Project Manager and one Senior Developer. Three experts worked at small organisations (between 6 and 49 employees, as defined in [28]), and one expert worked at a medium-sized organisation (between 50 to 249 employees). The average size of the teams appears to be small with no more than 8 members.

All of the experts had been working with Agile for between three to five years. Moreover, all of the interviewees were in an iterative sprint to deliver a release in one week, except for P3 who had a deadline of two weeks.

TABLE I

EXPERTS' OVERVIEW

\begin{tabular}{|l|l|l|l|l|}
\hline Position & P1 & P2 & P3 & P4 \\
\hline Company & CEO & CTO & $\begin{array}{l}\text { Project } \\
\text { Manager }\end{array}$ & $\begin{array}{l}\text { Senior } \\
\text { developer }\end{array}$ \\
\hline Company Size & A & B & C & D \\
\hline No. of Teams & 3 & Small & Medium & Small \\
\hline Average Team size & 7 & 1 & 2 & 1 \\
\hline Agile Method & Scrum & Scrum & Scrum & Scrum \\
\hline $\begin{array}{l}\text { Experiences in Mo- } \\
\text { bile Development }\end{array}$ & 11 years & 8 years & 10 years & 5 years \\
\hline $\begin{array}{l}\text { Experiences in Ag- } \\
\text { ile }\end{array}$ & 5 years & 4 years & 4 years & 3 years \\
\hline
\end{tabular}

\section{B. Awareness of Agile}

1) Awareness Level: All of the experts stated that the level of Agile awareness among mobile app practitioners is still low compared to other software development such as web apps. Therefore, they had only begun adopting Agile in their development process between one to three years prior to the interviews taking place, for instance, P2 stated he had adopted it just one year previously. Awareness of Agile is considered a critical issue facing software organisations in the country, as stared by $\mathrm{P} 1$ and $\mathrm{P} 4$. When asked about the reasons for the lack of awareness of Agile, P4 mentioned that the mobile app industry in Saudi Arabia is a young sector and there are still few experts in this domain compared to other software domains. Thus, most of the developers who adopt this approach could be considered novices. As stated by P1, who works as a CEO, "Most of the mobile app developers who start to work with us don't have knowledge about Agile methods, and the reason, I think, behind that is the lack of training events related to Agile in the country".

2) Knowledge Source: P2 stated that "the lack of awareness of Agile among developers is not a big issue with us". $\mathrm{He}$ mentioned that most of the developers who had joined their company had learnt Agile 'on the job'; hence, they had found it easy to learn about Agile. P1 highlighted that computer science colleges in Saudi universities do not put much emphasis on Agile methodology in terms of teaching and training compared to other approaches like Waterfall. All of the interviewees also stated that training and practice are the most significant sources 
of gaining more knowledge about Agile. Indeed, P4 mentioned that he learnt about Agile from university courses in the US, while the other participants had learned from online courses, training, practice and certifications.

\section{Use of Agile}

1) Agile Current Usage: With regard to the use of Agile, $\mathrm{P} 2$, who had been working as an IOS developer for a number of years, highlighted that Agile was not widely used in mobile app development. He asserted that most practitioners he knew did not follow a specific methodology and often used both Waterfall and Agile at the same time. All of the experts rated the extent to which Agile is applied in mobile app development as being minimal, due to a general lack of awareness and knowledge of Agile among practitioners and business teams. From P3's point of view, Agile was generally applied more in small organisations compared to those of other sizes. In addition, although there was a general consensus among interviewees that awareness and knowledge of Agile across Saudi Arabia was limited, they did agree that a number of software organisations were beginning to use Agile.

2) Agile Adoption: Although there was a lack of awareness about Agile in the country, there are some organisations adopting Agile for its perceived benefits. As highlighted by all experts of how they started adopting Agile. P3 mentioned that app developers began using Agile as they had first noticed the benefits of using Agile on web app development in their companies. Others agreed and shared that their organisations adopted Agile after attending training events and realising the benefits organisations obtain from adopting Agile. Furthermore, all experts agreed that the competitive environment in the mobile app industry was another driver for their organisations adopting Agile.

3) Agile Methods: Based on the experts' responses as depicted in Table I, Scrum was found to be the most frequently used Agile method for mobile app development. However, some interviewees, like P1 and P3, stated they did not only use Scrum. P1 asserted that there are some Agile teams that have adopted the Kanban method with Scrum. Furthermore, some mobile app teams were using Scrum fully while others were using it only partially.

4) Agile Techniques: As stated in the previous section, some of the organisations, where full Scrum was used, adopted a wide range of Agile techniques (i.e. daily stand-up meetings, sprint planning meetings, sprint reviews, sprint retrospectives, burndown charts, user stories, product backlogs and sprint backlogs). On the other hand, some of the organisations, where Scrum was only partially adopted, were using fewer Agile techniques, such as daily stand-up meetings, sprint planning meetings, sprint reviews, user stories, and sprint backlogs.

\section{Perceptions of Agile}

1) Reasons for Adopting Agile: When asked about the reasons that encouraged the interviewees to adopt Agile, the main motivations included the flexibility of Agile to manage projects and team members and an increased customer satisfaction compared to other methodologies used. In addition, they asserted that using Agile increased their productivity and the morale of the team members. P2 stated that "when Agile was adopted one year ago, the team members were extremely happy with it as they were able to see what they did every week and receive feedback". All of the interviewees affirmed that the main reason for adopting Agile was to decrease the development cycle times as well as time to market.

2) Reasons for Not-Adopting Agile: Although the participants agreed that Agile had changed their development process for the better, they also listed several drawbacks of adopting Agile. One of the most important reason preventing Agile adoption in Saudi Arabia is the lack of documentation Agile is considered an emerging methodology in the country. P3 stated that some customers prefer documentation of their projects. In addition, organisation culture and the business teams' lack of technical knowledge of Agile were considered the most significant reasons for the non-adopting of Agile, as stated by $\mathrm{P} 4$.

\section{SURVEY FINDINGS}

This section presents the findings of the survey. In total, 31 questionnaires were completed and collected from practitioners working in different mobile app development teams and organisations in Saudi Arabia.

\section{A. Demographic Information}

The data in this section presents the demographic information related to the participants and their organisations. This information provided the researchers of this study with an understanding of the respondents' backgrounds, including their gender, age, position in their respective organisations, years' experience in mobile app development, number of employees in their organisations, number of development teams they work with, the average number of team members, platforms used for app development, and types of apps developed.

1) Participants' Information: Table II represents the demographic information of the survey's participants. Of the respondents, 29 out of 31 (about 94\%) were male and the remaining $6 \%$ were female. The largest group of respondents were aged between 31 to 39 and 23 to 30, accounting for 52\% and $42 \%$ respectively, and the remaining $6 \%$ were aged 40 and above. In terms of the participants' experiences in mobile app development, $48 \%$ of them had 1 to 4 years' experience, while $39 \%$ had 5 to 10 years' experience. As shown in the table, the respondents were working in different roles, with the majority working as developers $(46.4 \%)$. This was followed by project manager $(24.3 \%)$, other positions (13.5\%), designers $(8.1 \%)$, CEO (5.4\%) and CTO/CIO (2.7\%).

2) Work Environment: The respondents were working in software organisations of different sizes in Saudi Arabia. 45\% of them were working in small organisations, $32 \%$ in mediumsized organisations and $19 \%$ were working in large organisations, while $3 \%$ were working in micro organisations with 5 or fewer employees. This illustrates that three out of the four 
TABLE II

GENDER, AGE, WORK EXPERIENCE, AND ROLE OF PARTICIPANTS

\begin{tabular}{|c|c|c|c|c|}
\hline Question & $\begin{array}{l}\text { Answer } \\
\text { options }\end{array}$ & Frequency & $\begin{array}{l}\text { Percentage } \\
(\%)\end{array}$ & $\begin{array}{l}\text { Cumulative } \\
\text { Percent }\end{array}$ \\
\hline \multirow{2}{*}{ Gender } & Male & 29 & 93.55 & 93.5 \\
\hline & Female & 2 & 6.45 & 100.0 \\
\hline \multirow{4}{*}{ Age } & Below 23 years & 0 & 0.0 & 0.0 \\
\hline & $23-30$ years & 13 & 41.94 & 41.9 \\
\hline & $31-39$ years & 16 & 51.61 & 93.5 \\
\hline & Above 40 years & 2 & 6.45 & 100.0 \\
\hline \multirow{4}{*}{$\begin{array}{l}\text { Experiences } \\
\text { in mobile } \\
\text { development }\end{array}$} & Less than a year & 2 & 6.45 & 6.5 \\
\hline & $1-4$ years & 15 & 48.39 & 54.8 \\
\hline & $5-10$ years & 12 & 38.71 & 93.5 \\
\hline & $\begin{array}{l}\text { More than } 10 \\
\text { years }\end{array}$ & 2 & 6.45 & 100.0 \\
\hline \multirow{6}{*}{ Role } & $\mathrm{CEO}$ & 2 & 5.41 & 5.41 \\
\hline & $\mathrm{CIO} / \mathrm{CTO}$ & 1 & 2.7 & 8.11 \\
\hline & Project manager & 9 & 24.32 & 32.4 \\
\hline & Developer & 17 & 45.95 & 78.38 \\
\hline & Designer & 3 & 8.11 & 86.49 \\
\hline & Others & 5 & 13.51 & 100.0 \\
\hline
\end{tabular}

organisations were small and medium-sized enterprises. With regard to the number of development teams the participants are involved in, 55\% reported being involved in one working team, $10 \%$ in two working teams, $13 \%$ in three working teams and $22 \%$ in three or more working teams. The average number of members in a development team was 6 to $10(48 \%)$ or 1 to 5 members (39\%), and no team had more than 20 members.

3) App Platforms and Types: With regard to the types of platforms and apps used, the results show that the IOS platform was the most popular platform among the mobile app practitioners, as $50 \%$ of the respondents chose IOS. This was followed by the Android platform (39\%). In addition, most of the participants $(51 \%)$ chose Native apps as the most common app type used, followed by Hybrid apps (29\%) and Web apps $(21 \%)$

\section{B. Software Development Information}

This section presents the findings from the second part of the survey, which contains items on the participants' experience working in software development, as well as the most popular software development methodologies used by mobile app practitioners. Most of the respondents (43\%) had 1 to 5 years work experience in software development, while $40 \%$ had between five to ten years' experience. In addition, $10 \%$ of the respondents had more than ten years' experience and $7 \%$ of participants had been working for less than a year.

1) Software Development Methodology: The second question measured the participants' knowledge about the software development methodologies, such as Waterfall, Spiral, Rapid Application, V, Agile and others, that they had used during their development process [29]. From the results, it is evident that the Waterfall model is the most commonly used software methodology (40\%), followed by Agile software development (29\%). As more than one methodology can be used, some participants selected more than one methodology. For the respondents who answered 'other' (23\%), this indicated that they did not use any specific methodology, while others used a combination of Waterfall and Agile. Moreover, no respondent selected the Spiral model and only $2 \%$ selected the V model.

\section{Agile Software Development Information}

This section contains items on Agile software development and practitioner awareness, usage and perceptions of it.

1) Awareness of Agile:

Awareness Level. Figure 1 presents the findings related to the participants' level of awareness of the use of Agile in mobile app development. The survey found that the vast majority of participants $(77 \%)$ had heard of Agile. That is, $33 \%$ of the them have limited knowledge, while another 33\% had a good awareness, and only a small number of respondents (10\%) had extensive knowledge. On the other hand, 23\% of them had never heard of Agile and were unaware of its existence. Most respondents had either less than a year or 1 to 4 years' experience using Agile. While several had more than 5 years' experience using Agile, none had more than 10 years' experience.

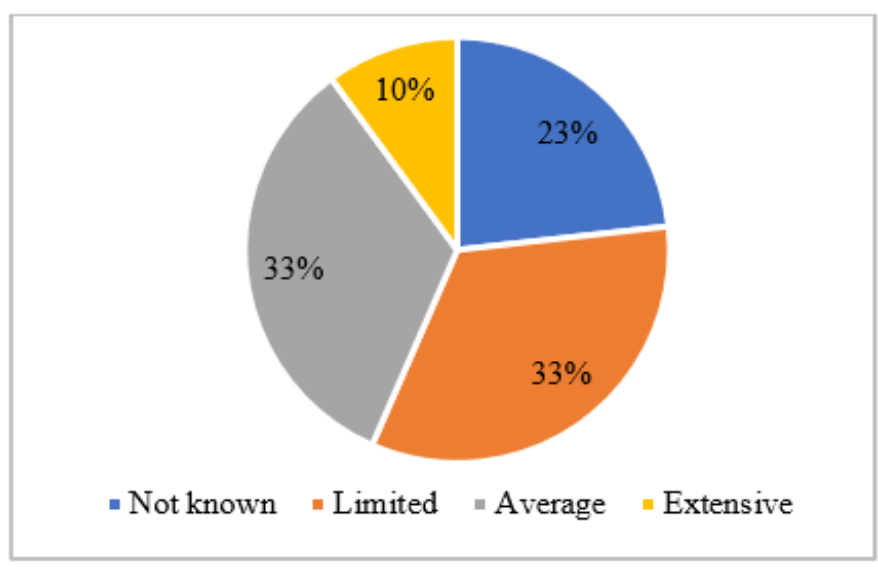

Fig. 1. Awareness of Agile.

Knowledge Source. The participants were also asked about how they obtain knowledge about Agile. Practice and reading were the most popular sources of knowledge among mobile app practitioners, with $39 \%$ and $35 \%$, respectively. Furthermore, $20 \%$ of them stated they attend training events and $6 \%$ prefer to take part in Agile certification. When participants asked about the importance of training team members to use Agile before applying it, over three quarters of them (77\%) agreed that training team members is "very important and important' and $14 \%$ described that training as being 'natural'. On the other hand, $9 \%$ of the respondents said that they thought that training is 'slightly important'. 


\section{2) Use of Agile:}

Agile Current Usage. Just under a quarter (22\%) of the participants responded that they fully adopted Agile methodology during the mobile app development process, while $61 \%$ of them stated they partially adopted Agile during the development process. The remaining $17 \%$ indicated they did not adopt Agile. A majority of the participants (70\%) stated that their organisations had not used Agile software development, whereas $30 \%$ of them had adopted Agile during the development process of mobile apps, and approximately half of them had been using Agile for 1 to 2 years.

$41 \%$ of the participants shared that their customers were happy with the use of Agile in their development process while half of them said that they are partially happy. However, $9 \%$ of them stated that their customers were not happy. Furthermore, the study found that $65 \%$ of the respondents think that there is a low use of Agile in Saudi Arabia, while 30\% of them answered that they felt neutral and 5\% agreed that Agile was applied in the Saudi mobile app development industry.

Agile Methods. A number of Agile methods were raised to figure out what Agile method has widely been used by the respondents. Hence, according to the data shown in Figure 2, $48 \%$ of participants used Scrum, while others used Kanban (15\%), Extreme Programming (12\%) and Crystal (12\%). These results are aligned with the results of other studies discussed in the related work section.

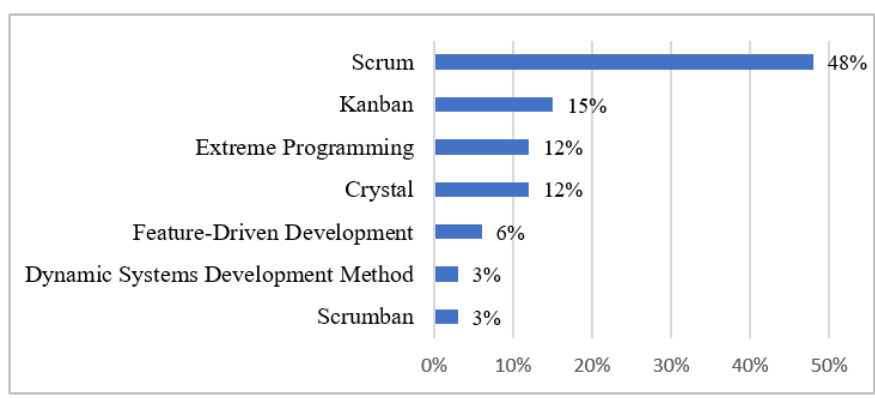

Fig. 2. Agile methods.

Agile Techniques. The respondents were asked whether they are aware of Agile and the extent to which they use Agile techniques. Table III shows the frequency of responses for each technique, as well as the number of respondents using each one, and the number of those who claim to know nothing about Agile. These techniques are ordered based on their usage, from the most to the least used. The study found that the most frequently used practices were 'user stories' and 'daily stand-up meeting', followed by 'sprint review', 'iterative development', and 'incremental development'. The practices which were the least popular were 'burndown chart' and 'sprint retrospectives'. However, even though some participants had never heard of Agile or used Agile, they indicated that they did use some Agile techniques, such as 'user stories' and 'daily stand-up meeting'. Moreover, when the respondents were asked about the tools they used in their mobile-Agile team, they responded that GitHub was the most used platform
(36\%) to manage the source code, followed by Bitbucket. Furthermore, 34\% of them used Asana and Jira for project management, while some of the respondents stated they did not use any tools. With regard to communication tools, they stated that Zoom and Slack are the most popular platforms used.

TABLE III

AgILE TECHNIQUES USAGE

\begin{tabular}{|l|c|c|c|} 
Techniques & Frequently & Percentage (\%) & \#NK. \\
\hline User stories & 20 & 14.93 & 4 \\
Daily stand-up meeting & 15 & 11.19 & 4 \\
Sprint review & 13 & 9.70 & 0 \\
Iterative development & 12 & 8.96 & 2 \\
Incremental & 12 & 8.96 & 2 \\
development & 11 & 8.21 & 2 \\
Product backlog & 11 & 8.21 & 0 \\
Sprint backlog & 11 & 8.21 & 0 \\
Sprint planning meeting & 8 & 5.97 & 2 \\
Test-driven development & 8 & 5.97 & 1 \\
Pair programming & 7 & 5.22 & 0 \\
Sprint retrospectives & 3 & 2.24 & 2 \\
Burndown chart & 3 & 2.24 & 0 \\
Others & &
\end{tabular}

\section{3) Perceptions of Agile :}

Reasons for Adopting Agile. The participants were asked to rank reasons for adopting Agile using a Likert Scale. The items were ranked based on Strongly Agree and Agree. As illustrated in Figure 7, 'improve team morale' and 'enhance ability to adapt to changes' were the main reasons for the encouragement of Agile adoption. 'Increase team productivity' was also one of the top reasons selected, with more than $80 \%$ of the respondents strongly agreeing with the statement. However, in order to 'reduce application development cost' was considered by the respondents as the least important reason.

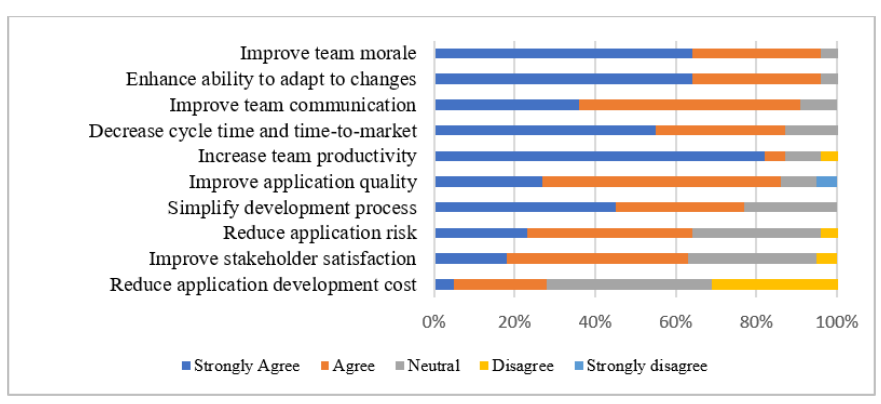

Fig. 3. Reasons for adopting Agile.

Reasons for Not-Adopting Agile. Figure 4 illustrates the respondents' reasons for not adopting Agile. The items were ranked based on Strongly Agree and Agree. The study found that 'lack of knowledge and training' was the most common 
reason (90\%). 'Traditional organisational culture' and 'the difficulty of defining business value' were the second and third reasons for preventing Agile adoption. On the other hand, the least important reasons for the non-adoption of Agile were 'Lack of tools to support adoption of Agile methods' and 'Application development is not suitable for Agile'.

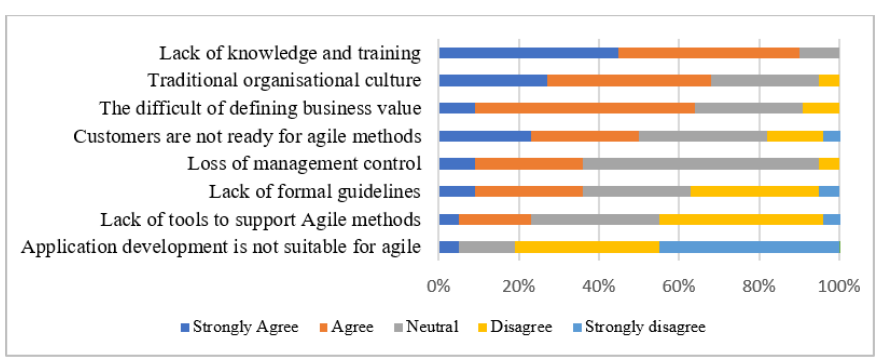

Fig. 4. Reasons for not-adopting Agile.

Agile Values. All of the participants were required to answer a question about the extent to which the four Agile values [3] were applied by their development teams. This presents the level of agility of the participants' teams even though they had never heard of Agile or used Agile. The results presented in Figure 5 indicate that most of the participants either strongly applied or applied the Agile values, except the value 'Customer collaboration over contract negotiation', which showed the highest neutral responses. Taking into consideration the total percentage of Strongly applied and Applied, it can be seen that the most popular value was 'Responding to change over following a plan', which represents about $76 \%$ of the responses. This is followed by 'Individuals and interactions over processes and tools', with around 59\%.

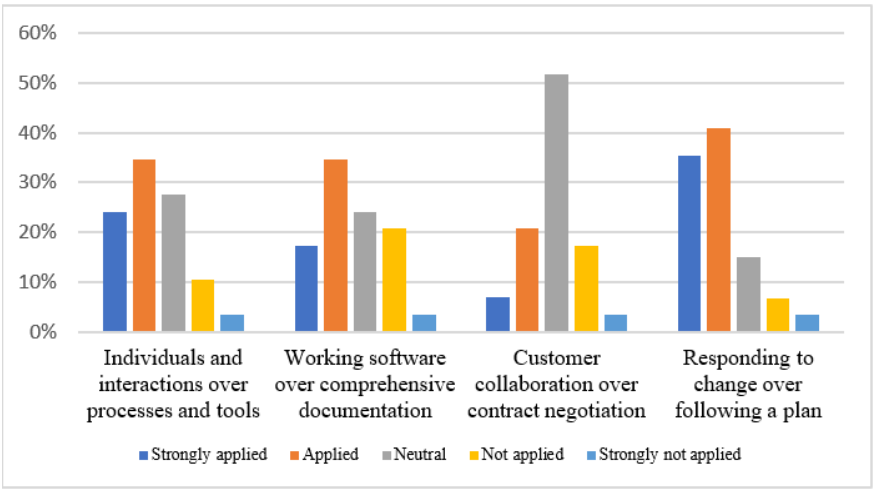

Fig. 5. Agile values.

\section{DISCUSSION}

The main focus of this paper is the level of awareness, current usage and perceptions of Agile methodology among mobile app practitioners in Saudi Arabia. This section discusses the significant findings derived from the interviews and the survey conducted in this study.

\section{A. Awareness of Agile}

Agile is a well-known methodology and is used by a number of companies in western countries [7], [8]. However, based on the data collected in this study, Agile is still not widely known in Saudi Arabia, and there is a low level of awareness of Agile amongst mobile app practitioners in the country. These results are similar to those reported in studies conducted in developing countries, such as Bin-Hezam et al. [21], Sulaiman et al. [12], and Nazir et al. [11]. Moreover, about a quarter of the survey respondents had never heard of Agile, whereas three quarters of them had heard about it with different levels of expertise.

The empirical results of this study suggest that the respondents have a limited knowledge of Agile. Therefore, including Agile courses in the university curriculum or conducting training events could help improve the awareness and perception of Agile in the country. This is a conclusion also drawn by de O. Melo et al. (2013) [10] based on the results of their Brazilian study. In order to encourage software practitioners or organisations to adopt Agile methods, raising awareness of it is considered as an initial step, since, due to the lack of awareness among mobile app practitioners, only a small number of organisations have adopted Agile into the process of mobile app development.

\section{B. Use of Agile}

As Agile awareness is low, the usage of it is not widespread in the country as evidenced by the results of this study, showing that $30 \%$ of the organisations have adopted Agile. However, the early adopters of Agile in the study indicate that adopting Agile methods is highly applicable to develop a mobile app and has a positive impact on producing highquality apps and reducing the time-to-market. Despite these advantages, Agile has only been applied fully by a minority of organisations in the country. This is due to the low awareness and knowledge the participants have, which these are considered as initial steps to implement Agile methods properly. This study indicates that there is a low level of Agile usage in Saudi Arabia, and that contrasted with the earlier finding of earlier worldwide survey study conducted by VersionOne [30] that reported that $82 \%$ of respondents was using Agile.

The results show that the most common Agile method adopted was Scrum, which is also considered the most popular method for most of the studies in the literature (e.g. Nazir, Hasteer and Bansal [11]; Melo et al. [10]; the VersionOne report [30]). This finding broadly supports the work of other studies that were conducted in western countries such as [7]. However, other studies, for example, the study by Salo and Abrahamson [9] found that their respondents are more familiar with XP than Scrum.

This study has found that the most frequently used Agile practices among software practitioners in Saudi Arabia are 'user stories' and 'daily stand-up meeting'. In addition, the most interesting finding was that these two techniques are the most citied by the respondents who were not aware of Agile or had never used it before. Whereas 'burndown chart' and 'sprint retrospectives' are cited as the least used 
techniques in our study, they are opposed to the findings of the study by VersionOne [30], which indicated that one of the most widely employed techniques was retrospectives. Another interesting finding from the current study is that respondents have indicated they are applying some Agile values such as 'Responding to change over following a plan'. Hence, even though some respondents claim to have no knowledge of Agile, they might be adopting some Agile techniques and applying some Agile values.

\section{Perceptions of Agile}

A number of studies have indicated the importance of Agile in the software industry, as reported in the literature. One of the major benefits of Agile is its ability to respond to changes in customer requirements, as mentioned in the Agile Manifesto [3]. This was echoed by the Agile experts interviewed and the respondents of the survey in this study. In addition, this research has found that improved team morale, communication and productivity are perceived as key benefits; this was also reported by Begel and Nagappen [8] and Rodriguez et al. [7]. The flexibility of Agile to manage projects and the increase of customer satisfaction are stated as reasons for adopting Agile by the early adopters. These benefits have been stated in previous studies as main reasons for adopting Agile [7], [20], [30].

On the other hand, the results of this study show that 'Lack of knowledge and training', 'Lack of documentation', and 'Traditional organisational culture' were the top reasons for preventing adopted Agile; these findings are not dissimilar to studies conducted in both Brazilian and Thai contexts [10], [20]. Practitioners should adapt how Agile works and obtain the relevant knowledge of Agile methods in order to adopt Agile in their development process. Therefore, more learning and education are required in the country to reduce the challenges of adopting Agile. The experts interviewed stated that the most helpful way to learn Agile is by training and practice.

Hence, awareness and knowledge, organisational culture, training and learning, customer satisfaction and communication are considered as factors that influence Agile adoption, as these factors have been identified as critical success factors of adopting Agile in [31]-[33]. These factors will be investigated in-depth in the Saudi software industry through empirical research in future work.

\section{CONCLUSION AND FUTURE WORK}

The goal of this study was to investigate the awareness, current usage and perceptions of Agile software development methodology among mobile app practitioners in Saudi Arabia. This study gathered data from semi-structured interviews with four experts and a survey completed by 31 participants. Thus, this study contributes greater knowledge of Agile among software practitioners and organisations in Saudi Arabia and adds to the body of knowledge on Agile implementation in Middle Eastern countries. This study concludes that there is currently little awareness of Agile among practitioners in Saudi
Arabia, as its implementation is in the early stages in the country. However, it also finds that several practitioners do actually apply certain Agile techniques and principles, even without having heard of Agile. There is also an indication from the experts interviews and some of the survey responses that Agile could be the best methodology for developing mobile applications.

Limitations - This study has been carried out with a limited sample size, which may limit the generalizability of the results. However, the study protocol was rigorously constructed thus its findings are robust, and the protocol can be more widely adopted in future studies. Most importantly, it offers a first insights into an emerging and significant area of research.

Future research should investigate the awareness, current usage and perceptions of Agile from other perspectives, such as customers, developers, project managers. Furthermore, future work could investigate Agile in different development contexts such as web app development or software development in general in Saudi Arabia. This work provides a 'birds-eye-view' of this emerging research area across different organisations, future research may focus on specific case studies to gain a deeper insight into the use of Agile in different and more specific contexts.

This study is an initial step in investigating the factors influencing Agile adoption in the Saudi Arabian mobile app development context [34]. We are revising these factors obtain from this study and the literature. Our future works will empirically investigate these factors among mobile app practitioners in the context of software development SMEs in Saudi Arabia using semi-structured interviews and focus groups.

\section{ACKNOWLEDGMENT}

We would like to thank all of the participants for participating in this study.

\section{REFERENCES}

[1] G. L. Urban, T. Carter, S. Gaskin, and Z. Mucha, "Market Share Rewards to Pioneering Brands: An Empirical Analysis and Strategic Implications," Management Science, vol. 32, pp. 645-659, jun 1986.

[2] Ian Sommerville, "Agile software development," in Software Engineering, ch. 3, pp. 40-70, Boston, US: Pearson Education, Addison-Wesley, 10th ed., 2016

[3] K. Beck, M. Beedle, A. van Bennekum, A. Cockburn, W. Cunningham, M. Fowler, J. Grenning, J. Highsmith, A. Hunt, R. Jeffries, J. Kern, B. Marick, R. C. Martin, S. Mellor, K. Schwaber, J. Sutherland, and D. Thomas, "Manifesto for Agile Software Development," 2001.

[4] E. Winter, S. Forshaw, and M. A. Ferrario, "Measuring human values in software engineering," in Proceedings of the 12th ACM/IEEE International Symposium on Empirical Software Engineering and Measurement, pp. 1-4, 2018.

[5] E. Winter, S. Forshaw, L. Hunt, and M. A. Ferrario, "Advancing the study of human values in software engineering," in 2019 IEEE/ACM 12th International Workshop on Cooperative and Human Aspects of Software Engineering (CHASE), pp. 19-26, IEEE, 2019.

[6] A. Santos, J. Kroll, A. Sales, and P. Fernandes, "Investigating the Adoption of Agile Practices in Mobile Application Development," in Proceedings of the 18th International Conference on Enterprise Information Systems, pp. 490-497, 2016.

[7] P. Rodríguez, J. Markkula, M. Oivo, and K. Turula, "Survey on agile and lean usage in finnish software industry," in International IEEE Symposium on Empirical Software Engineering and Measurement, pp. 139148, 2012. 
[8] A. Begel and N. Nagappan, "Usage and Perceptions of Agile Software Development in an Industrial Context: An Exploratory Study," in The First IEEE International Symposium on Empirical Software Engineering and Measurement (ESEM), pp. 255-264, 2007.

[9] O. Salo and P. Abrahamsson, "Agile methods in European embedded software development organisations: A survey on the actual use and usefulness of Extreme Programming and Scrum," IET Software, vol. 2, no. 1, pp. 58-64, 2008.

[10] C. de O. Melo, V. Santos, E. Katayama, H. Corbucci, R. Prikladnicki, A. Goldman, and F. Kon, "The evolution of agile software development in Brazil: Education, research, and the state-of-the-practice," Journal of the Brazilian Computer Society, vol. 19, no. 4, pp. 523-552, 2013.

[11] N. Nazir, N. Hasteer, and A. Bansal, "A survey on agile practices in the Indian IT industry," in Proceedings of the 2016 6th International Conference - Cloud System and Big Data Engineering, Confluence 2016 , pp. 635-640, 2016.

[12] N. L. Sulaiman, M. N. R. Mahrin, and R. Che Mohd Yusoff, "Investigating awareness issues with Agile practices," in 9th IEEE Malaysian Software Engineering Conference, MySEC, pp. 183-188, 2015.

[13] L. Corral, A. Sillitti, and G. Succi, "Software development processes for mobile systems: Is agile really taking over the business?," in 2013 IEEE 1st International Workshop on the Engineering of Mobile-Enabled Systems (MOBS), pp. 19-24, IEEE, 2013.

[14] S. B. Kaleel and S. Harishankar, "Applying agile methodology in mobile software engineering: Android application development and its challenges," Computer Science Technical Reports, pp. 1-11, 2013.

[15] T. Wasserman, "Software engineering issues for mobile application development," in Proceedings of the ACM Workshop on the Future of Software Engineering Research, FoSER, pp. 397-400, 2010.

[16] M. R. Salinas, A. G. S. Neto, and M. C. F. Emer, "Concerns and limitations in agile software development: A survey with paraguayan companies," in Communications in Computer and Information Science, vol. 802, pp. 77-87, Springer Verlag, 2018.

[17] A. Solinski and K. Petersen, "Prioritizing agile benefits and limitations in relation to practice usage," Software Quality Journal, vol. 24, no. 2 , pp. 447-482, 2016.

[18] P. Serrador and J. K. Pinto, "Does Agile work? - A quantitative analysis of agile project success," International Journal of Project Management, vol. 33, no. 5, pp. 1040-1051, 2015.

[19] M. Pikkarainen, O. Salo, R. Kuusela, and P. Abrahamsson, "Strengths and barriers behind the successful agile deployment-insights from the three software intensive companies in Finland," Empirical Software Engineering, vol. 17, pp. 675-702, dec 2012.

[20] A. Nanthaamornphong and R. Wetprasit, "A case study: Adoption of agile in Thailand," in 2016 IEEE International Conference on Advanced Computer Science and Information Systems, ICACSIS 2016, pp. 585590, IEEE, 2016.

[21] R. Bin-Hezam, A. Bin-Essa, and N. F. Abubacker, "Is the Agile Development Method the Way to Go for Small to Medium Enterprises (SMEs) in Saudi Arabia?," in 21st IEEE Saudi Computer Society National Computer Conference, NCC 2018, pp. 1-6, IEEE, 2018.

[22] J. W. Creswell and J. D. Creswell, Research design: Qualitative, quantitative, and mixed methods approaches. CA, USA: Sage publications, 5th ed., 2018.

[23] G. Biesta, A. Tashakkori, and C. Teddlie, SAGE Handbook of Mixed Methods in Social \& Behavioral Research. Los Angeles, CA: Sage publications, 2ed ed., 2010.

[24] R. Boyatzis, Thematic Analysis and Code Development. Sage Publications, Inc., 1998.

[25] V. Braun and V. Clarke, "Thematic analysis," in APA handbook of research methods in psychology, Vol. 2: Research designs: Quantitative, qualitative, neuropsychological, and biological (H. Cooper, P. M. Camic, D. L. Long, A. T. Panter, D. Rindskopf, and K. J. Sher, eds.), pp. 57-71, Washington, DC: American Psychological Association, 2012.

[26] T. P. Johnson, "Snowball Sampling: Introduction," in Wiley StatsRef: Statistics Reference Online (N. Balakrishnan, T. Colton, B. Everitt, W. Piegorsch, F. Ruggeri, and J. L. Eugels, eds.), John Wiley \& Sons, Ltd, sep 2014.

[27] A. Field, Discovering statistics using IBM SPSS statistics. CA, USA: Sage publications, 4th ed., 2013.

[28] M. of Commerce, "The Board of Directors of the General Authority for Small \& Medium Enterprises Specifies the Definition of Micro, small and Medium Enterprises," 2016.
[29] Dean Leffingwell, Agile Software Requirements: Lean Requirements Practices for Teams, Programs, and the Enterprise. Addison-Wesley Professional, 2010.

[30] V. One, "The 14th annual State of Agile Report," tech. rep., 2020.

[31] J. Sheffield and J. Lemétayer, "Factors associated with the software development agility of successful projects," International Journal of Project Management, vol. 31, no. 3, pp. 459-472, 2013.

[32] T. Chow and D. B. Cao, "A survey study of critical success factors in agile software projects," Journal of systems and software, vol. 81, no. 6, pp. 961-971, 2008.

[33] S. C. Misra, V. Kumar, and U. Kumar, "Identifying some important success factors in adopting agile software development practices," Journal of Systems and Software, vol. 82, no. 11, pp. 1869-1890, 2009.

[34] F. S. Altuwaijri and M. A. Ferrario, "Investigating Agile Adoption in Saudi Arabian Mobile Application Development," in Agile Processes in Software Engineering and Extreme Programming - Workshops, XP2020 (M. Paasivaara and P. Kruchten, eds.), (Cham), pp. 265-271, Springer, 2020. 